\title{
A nanoflare heating model for the quiet solar corona
}

\author{
U. Mitra-Kraev and A. O. Benz \\ Institute of Astronomy, ETH-Zentrum, 8092 Zürich, Switzerland \\ e-mail: benz@astro.phys.ethz.ch; urmila@astro.phys.ethz.ch \\ Received 29 January 2001 / Accepted 4 April 2001

\begin{abstract}
The energy input into the lower solar corona by flare evaporation events has been modeled according to the available observations for quiet regions. The question is addressed whether such heating events can provide the observed average level of the coronal emission measure and thus of the observed flux of extreme ultraviolet (EUV) and X-ray emission without contradicting the observed average power spectrum of the emission measure, the typical emission measure variations observed for individual pixels and the observed flare energy distribution. As the assumed flare height influences the derived flare energy, the mathematical foundations of nanoflare distributions and their conversion to different height assumptions are studied first. This also allows a comparison with various published energy distributions differing in height assumptions and to relate the observations to the input parameters of the heating model. An analytic evaluation of the power spectrum yields the relationship between the average time profile of nanoflares (or microflares), assumed to be self-similar in energy, and the power spectrum. We find that the power spectrum is very sensitive to the chosen time profile of the flares. Models are found by numerical simulation that fit all available observations. They are not unique but severely constrained. We concentrate on a model with a flare height proportional to the square root of the flare area. The existence of a fitting model demonstrates that nanoflare heating of the corona is a viable and attractive mechanism.
\end{abstract}

Key words. Sun: corona - Sun: flares - Sun: transition region - Sun: chromosphere - Sun: UV radiation Sun: activity

\section{Introduction}

Many mechanisms have been proposed in the past for heating the solar corona (e.g. Ulmschneider et al. 1991). Most popular at present are models based on either the release of magnetic energy or the dissipation of waves. Both scenarios were proposed soon after the discovery of the high coronal temperature by Edlén and Grotrian in the late 1930s. As the magnetic energy dominates the coronal energy density, the release of free magnetic energy, possibly present in the corona in the form of electric currents, is a very suggestive idea. However, it was soon realized that the electric resistivity caused by collisions between electrons and ions is extremely low in the thin corona, making this mechanism insufficient. Therefore, several wave heating mechanisms have been proposed. Most recent theories involve Alfvén waves which can explain both the preferred heating of regions with high magnetic fields and solar wind acceleration (e.g. Marsh \& Tu 1997).

Gold (1964) seems to have been the first to suggest that the building up of free magnetic energy in the corona must be dissipated by flares when the resistivity becomes finite

Send offprint requests to: A. O. Benz,

e-mail: benz@astro.phys.ethz.ch by some instability. The idea was further developed by Levine (1974), Parker (1983), Heyvaerts \& Priest (1984) and others. Cargill (1994) pointed out that impulsively heated loops would cool by conduction and radiation with observable results. The general view of these studies was confined to the problem of releasing magnetic energy in the corona with the goal to keep it hot.

This is not supported by recent observations. Deep exposures of the soft X-ray emission in a quiet region by Yohkoh/SXT revealed that the emission measure of the corona in certain pixels was not constant over the observing time of about one hour (Krucker et al. 1997). It suggests that the material content of the low corona, where most of the soft X-ray emission originates, varies. More sensitive observations of EUV lines from the corona show variability of the majority of pixels $(85 \%)$, including some in the faint intra-cell regions (Benz \& Krucker 1998; Berghmans et al. 1998). The increases of the emission measure are not adiabatic compressions and can only be interpreted as additions of new material into the corona. The heated material subsequently seems to cool on a time scale of the order of $15 \mathrm{~min}$. Thus the coronal material in the lower corona appears to be not heated, but rather continuously replaced. We may add here, however, that 
a heating process could still be hidden in the quasistationary background of the emission measure.

Some large events have recently been tested by Brown et al. (2000) for their relation between increases in emission measure and temperature. The authors conclude that this relation corresponds more to an impulsive heating of the upper chromosphere and subsequent expansion rather than a heating at the top of a coronal loop followed by conductive readjustment. Thus the observed increases of the coronal emission measure may be interpreted as "chromospheric evaporation" similar to regular flares in active regions. Benz \& Krucker (1999) and Krucker \& Benz (2000) find very little difference between flares and the observable properties of the emission measure increases, and thus confirm the latter as real nanoflares. In the following, we will use the term nanoflare, first introduced by Parker (1983) as a theoretical concept, for any brightening of the quiet corona with energy below approximately $10^{26} \mathrm{erg}$.

Krucker \& Benz (1998) estimated the total energy input by the emission measure increases to be $16 \%$ of the calculated total radiative loss of the observed region. This number depends strongly on the sensitivity of the instrument and some model parameters. In particular, the effective line-of-sight thickness of the coronal plasma (or height for observations in the center of the disk) cannot be measured and must be assumed. The distribution of the events in energy is therefore still controversial. Most observers report a power law shape, but widely disagree on the exponent, which ranges from -1.45 (Berghmans \& Clette 1999, measuring radiation loss in Fe XII) to -2.59 (Krucker \& Benz 1998, measuring emission measure increases). Benz \& Krucker (2001a) reported agreement between their EIT based analysis and studies based on TRACE data by Parnell \& Jupp (2000) and Aschwanden et al. (2000), if the same method is used. For a flare model with a height proportional to the square root of the flare area, a simultaneous peak time within 2 min over the flare area and no further flare selection, all three investigations yield a power law index in the range between -2.0 and -2.4 , the most likely new EIT value being -2.3 . Clearly, nanoflare heating of the quiet corona is strongly supported by the above observations on total energy input and the relatively steep slope of the energy distribution, which suggests that the smallest flares contribute most to the heating.

Here we address the question of whether nanoflares can account for all of the observed properties of the emission measure in the quiet corona, including (i) the general appearance of individual pixels' emission measure time dependence, including background and nanoflares, (ii) the absolute value of the quasi-steady emission measure (equivalent to the total radiative loss), and (iii) the average Fourier spectrum of the emission measure in time, reported to be a power law with an exponent of -1.76 (Benz \& Krucker 1998).

First, we derive the conversion of flare frequency distributions in energy for different assumptions, in particular the conversion between distributions of flares, where pixels have been grouped into events, and distributions of single pixels, as well as the conversion between different height models (Sect. 2). In Sect. 3 we simulate the emission measure fluctuations in the quiet corona by taking the observed flare frequency distribution in energy, making certain assumptions on the spatial and temporal shape of the events and extrapolating them to the smallest energies needed to explain the total radiation loss from the corona. By choosing an adequate time profile for each flare, we can simulate the time dependence of the emission measure for an arbitrary pixel. We also obtain an averaged power spectrum. In Sect. 4 we calculate the expected value of the power spectrum analytically and in Sect. 5 the results are discussed. Section 6 summarizes the conditions needed for the nanoflare model to reproduce the observed features of the variations and quasi-steady background of the quiet corona.

\section{Flare distributions}

The observations that have been made of the quiet Sun to determine the energy input into the corona are based on a grid of many pixels in a large field of view. For each pixel and time interval, the emission measure is estimated (cf. Benz \& Krucker 1998; Parnell \& Jupp 2000). Neighboring and simultaneously occurring events are combined and interpreted as one flare. The thermal energy of an emission measure increase can be estimated (Benz \& Krucker 1998)

$E \approx 3 k_{\mathrm{B}} T \sqrt{\triangle \mathcal{M A h}}$

where the emission measure increase $\Delta \mathcal{M}$, the flare area $A$ and the temperature $T$ are observed quantities and $k_{\mathrm{B}}$ is the Boltzmann constant. Equation (1) assumes that the newly injected material is much denser than the pre-flare density in the loop. As pointed out by Brown et al. (2000), this assumption yields an upper limit to the energy. On the other hand, the flare energy release may also directly heat the coronal material, whose energy is not included in Eq. (1). In the following, we will use Eq. (1) as a rough estimate for the thermal energy input by a nanoflare. The height (thickness) $h$ of the flare loop lies in the line of sight and is therefore not easy to determine. We will use it here as a free model parameter that also includes the filling factor. Thus $h$ denotes the effective height.

All observers agree that the flare frequency distribution function depends on the energy as a power law

$f(E)=f_{0} E^{-\delta}$

where the flare distribution is defined as the number of flare events within a given energy interval divided by the total observed area, total observation time and energy interval. Similarly, the flare distribution in area was reported to be a power law (Aschwanden et al. 2000; Alietti et al. 2000). Supported by these observed distributions, it is assumed in the following that flares are self-similar in thermal energy. Thus on average, a flare is completely characterized by its energy. This assumption will later be tested against observations. 
Let us therefore write the emission measure increase, the flare area and the flare height, each averaged for a given thermal energy, in terms of some power of the energy

$$
\begin{aligned}
\Delta \mathcal{M} & =c \cdot E^{\gamma} \\
A & =a \cdot E^{\alpha} \\
h & =b \cdot E^{\beta} .
\end{aligned}
$$

Where no confusion is possible, we will use the same symbols for averaged entities, such as $\Delta \mathcal{M}$, and measured quantities in individual events. Equations (3)-(5) together with Eq. (1) imply the two conditions

$$
\begin{aligned}
\alpha+\beta+\gamma & =2 \\
a b c\left(3 k_{\mathrm{B}} T\right)^{2} & =1 .
\end{aligned}
$$

It is seen immediately from Eq. (1) that the energy of a flare depends on the height assumption, and so do the parameters $f_{0}$ and $\delta$ in Eq. (2). In the first and second part of this section we study the scaling relations and conversions between flare energy distributions for different assumptions for $h$ as well as for time variations of individual pixels. In the third part of this section we will apply these relations to numerical values from observations.

\subsection{Distribution in flare area and per pixel}

Because both the emission measure increase and the area of a flare are observable quantities, we write

$\Delta \mathcal{M}(A)=m A^{\mu}$.

In terms of the above variables we have

$$
\begin{aligned}
\mu & =\frac{\gamma}{\alpha} \\
m & =c \cdot a^{-\mu} .
\end{aligned}
$$

There are four equations (Eqs. (6), (7), (9) and (10)) and six variables $(a, b, c, \alpha, \beta, \gamma)$, thus the system is still not fully determined. This is because we have the freedom to make an assumption regarding the height dependence. A model for the flare height fixes $b$ and $\beta$, and the system of equations can be solved. There is one more difficulty, however, and that is to determine $\mu$ and $m$. As Eq. (8) is a direct relation between variables of individual events and not a distribution, the scatter is large, which is obvious in Fig. 1. There is another way to determine $\mu$ and $m$, using the comparison between flare distribution and the energy distribution of emission measure increases observed in individual pixels. In the following, we refer to the latter as pixel distribution and mark it with the index $p$.

The pixel distribution function accounts for events in pixels without adjacent pixels being grouped together to form one flare. Thus for flares covering more than one pixel, the pixel distribution is the flare distribution times the average flare area measured in units of pixel area $A_{\mathrm{p}}$

$f_{\mathrm{p}}\left(E_{\mathrm{p}}\right) \mathrm{d} E_{\mathrm{p}}=\frac{A(E)}{A_{\mathrm{p}}} f(E) \mathrm{d} E$, where the thermal energy of a pixel is $E_{\mathrm{p}}=$ $3 k_{\mathrm{B}} T \sqrt{\Delta \mathcal{M}_{\mathrm{p}} A_{\mathrm{p}} h}$. The average emission measure increase in a pixel is simply $\Delta \mathcal{M}_{\mathrm{p}}=A_{\mathrm{p}} / A \cdot \Delta \mathcal{M}$. It follows that

$E_{\mathrm{p}}=\frac{A_{\mathrm{p}}}{A(E)} E$.

We insert Eq. (4) into Eq. (12) and solve for $E$ to obtain an average relation between the thermal energy input in flares and in pixels

$E=\left(\frac{a}{A_{\mathrm{p}}} E_{\mathrm{p}}\right)^{\frac{1}{1-\alpha}}$.

Taking the derivative

$\frac{\mathrm{d} E}{\mathrm{~d} E_{\mathrm{p}}}=\frac{1}{1-\alpha}\left(\frac{a}{A_{\mathrm{p}}}\right)^{\frac{1}{1-\alpha}} E_{\mathrm{p}}^{\frac{\alpha}{1-\alpha}}$,

rewriting Eq. (11) as

$f_{\mathrm{p}}\left(E_{\mathrm{p}}\right) \mathrm{d} E_{\mathrm{p}}=\frac{A\left(E\left[E_{\mathrm{p}}\right]\right)}{A_{\mathrm{p}}} f\left(E\left[E_{\mathrm{p}}\right]\right) \frac{\mathrm{d} E}{\mathrm{~d} E_{\mathrm{p}}} \mathrm{d} E_{\mathrm{p}}$

and inserting Eqs. (2), (4), (13) and (14) into Eq. (15), leads to the final result for the relation between the flare distribution and the pixel distribution

$$
\begin{aligned}
f_{\mathrm{p}}\left(E_{\mathrm{p}}\right) & =\frac{1}{1-\alpha}\left(\frac{a}{A_{\mathrm{p}}}\right)^{\frac{2-\delta}{1-\alpha}} f_{0} E_{\mathrm{p}}^{-\frac{\delta-2 \alpha}{1-\alpha}} \\
& =: f_{\mathrm{p} 0} E_{\mathrm{p}}^{-\delta_{p}} .
\end{aligned}
$$

The exponent $\alpha$ and the factor $a$ then are

$\alpha=\frac{\delta_{\mathrm{p}}-\delta}{\delta_{\mathrm{p}}-2}$

$a=A_{\mathrm{p}}\left(\frac{f_{\mathrm{p} 0}}{f_{0}} \cdot \frac{\delta-2}{\delta_{\mathrm{p}}-2}\right)^{\frac{-1}{\delta_{\mathrm{p}}-2}}$.

Equation (6) together with the height assumption used to derive the thermal energy then yields $\gamma$, and $\mu$ follows immediately

$\mu=\frac{\gamma}{\alpha}=\frac{\delta_{\mathrm{p}}+\delta-4-\beta\left(\delta_{\mathrm{p}}-2\right)}{\delta_{\mathrm{p}}-\delta}$.

Similarly, one derives

$m=\frac{a^{-(1+\mu)}}{b\left(3 k_{\mathrm{B}} T\right)^{2}}$.

\subsection{Scaling relationships}

The primary free model parameter is the height of the flare (Eq. (5)), i.e. the parameters $b$ and $\beta$. They determine the thermal energy by Eq. (1) when $T, \Delta \mathcal{M}$ and $A$ are observed. In the following we derive the conversion between the flare frequency distributions of different height dependencies.

Let the new flare distribution be $f^{\prime}\left(E^{\prime}\right)$, where $E^{\prime}=$ $3 k_{\mathrm{B}} T \sqrt{\triangle \mathcal{M} A h^{\prime}}$. Then the conversion from the old flare distribution (Eq. 2) to the new one is given by

$f^{\prime}\left(E^{\prime}\right) \mathrm{d} E^{\prime}=f\left(E\left[E^{\prime}\right]\right) \frac{\mathrm{d} E}{\mathrm{~d} E^{\prime}} \mathrm{d} E^{\prime}$. 
It is seen immediately that the identity $E / \sqrt{h}=E^{\prime} / \sqrt{h^{\prime}}$ holds. Inserting Eq. (5), we obtain from it the condition

$b^{-\frac{1}{2}} E^{1-\frac{\beta}{2}}=\left(b^{\prime}\right)^{-\frac{1}{2}}\left(E^{\prime}\right)^{1-\frac{\beta^{\prime}}{2}}$.

Solving for $E$ leads to

$E\left(E^{\prime}\right)=\left(\frac{b}{b^{\prime}}\right)^{\frac{1}{2-\beta}} E^{\prime \frac{2-\beta^{\prime}}{2-\beta}}$.

The derivative is

$\frac{\mathrm{d} E}{\mathrm{~d} E^{\prime}}=\frac{2-\beta^{\prime}}{2-\beta}\left(\frac{b}{b^{\prime}}\right)^{\frac{1}{2-\beta}} E^{\prime \frac{\beta-\beta^{\prime}}{2-\beta}}$.

Inserting now Eqs. (2), (23) and (24) into Eq. (21) gives the new flare distribution

$$
\begin{aligned}
f^{\prime}\left(E^{\prime}\right) & =\frac{2-\beta^{\prime}}{2-\beta}\left(\frac{b}{b^{\prime}}\right)^{\frac{1-\delta}{2-\beta}} f_{0} E^{-\frac{\delta\left(2-\beta^{\prime}\right)-\beta+\beta^{\prime}}{2-\beta}} \\
& =: f_{0}^{\prime} E^{\prime-\delta^{\prime}}
\end{aligned}
$$

defining the new power law exponent $\delta^{\prime}$ and $f_{0}^{\prime}$.

\subsection{Numerical values}

The observed quantities are the emission measure, the flare area and the temperature. Benz \& Krucker (2001b) find an average temperature of $1.46 \times 10^{6} \mathrm{~K}$ for the 23 largest nanoflares that occurred in a quiet region within one hour. The temperature does not depend on the flare energy, and its distribution is narrow. The highest observed value was reported to be $T=1.63 \times 10^{6} \mathrm{~K}$. We use the former value for our models and will discuss it more in Sect. 5 . The pixel area is given by $A_{\mathrm{p}}=1900 \mathrm{~km} \times 1900 \mathrm{~km}$.

Making an assumption on the flare height, the flare energy, flare distribution, pixel energy and pixel distribution can be reduced, and thus also the parameters $m$ and $\mu$ defined in Eq. (8) according to Sect. 2.1. Once we know $m$ and $\mu$, we can solve for the parameters $a, b, c$ and $\alpha$, $\beta, \gamma$. Also, we can calculate a new flare distribution from Eq. (25) and a new pixel distribution from Eq. (16) for a different flare height. This shows the effect of the model assumption on the distribution and characteristic energies. Finally, the biggest interest is in the parameters for a model with flare height that scales with flare size.

Table 1 displays some parameters of interest. The values for $f_{0}$ and $\delta$ for $h=5000 \mathrm{~km}$ are from Krucker \& Benz (1998) and $f_{\mathrm{p} 0}$ and $\delta_{\mathrm{p}}$ for $h=500 \mathrm{~km}$ are from Krucker (private communication). All the other values from $f_{0}$ to $\gamma$ are calculated using the methods of the previous sections.

First, $f_{0}(500 \mathrm{~km})$ and $\delta(500 \mathrm{~km})$ have been calculated from Eq. (25), then $m$ and $\mu$ from Eqs. (20) and (19). The relation of Eq. (8) is displayed in Fig. 1 (solid line). This figure also contains the largest 22 events reported by Krucker \& Benz (2000) from a large field of view, thus the emission measures are relatively large (their Table 1, shown here by + ), and the largest 6 events from a smaller set (their Table 2 , shown by $*$ ). The calculated relation between emission measure and flare area matches well that

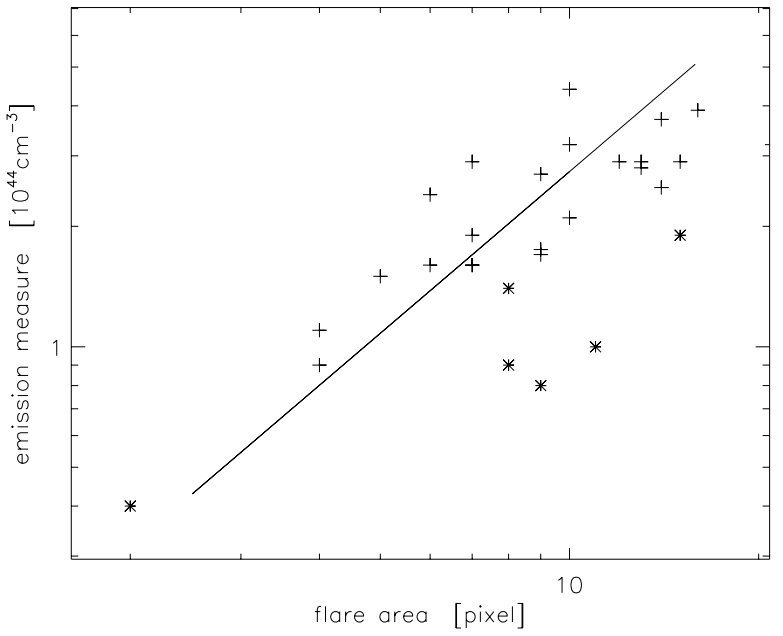

Fig. 1. Emission measure per flare versus flare area. The crosses are the largest flares observed in a large field of view and the asterisks refer to the largest events in a small field of view (Krucker \& Benz 2000). The solid line represents the relation $\Delta \mathcal{M}=m A^{\mu}$ (Eq. (8)), using the parameters calculated from Eqs. (19) and (20) for $\beta=0$.

observed for individual events. Knowing the parameters $m=7 \times 10^{20}$ and $\mu=1.34$, which are independent of the height model, all the other parameters can then immediately be calculated for any chosen height dependence.

Rather large nanoflares are seen to cover an area of approximately rectangular shape with a length to width ratio $q=l / w$ of about 10 . Thus

$A=l \cdot w \approx l^{2} / 10=10 w^{2}$.

As ordinary flares are believed to be generated in loopshaped magnetic field structures, nanoflares may be expected to originate in similar geometries. Thus, we assume the nanoflares to occur in torus-shaped loops with a loop-thickness equal to the flare width. In our approximation this loop-thickness shall just be the unknown effective height, thus $h=w=\sqrt{(A / 10)}$ and $\beta=\alpha / 2$ and $b=\sqrt{(a / 10)}$.

The largest single-peaked flare observed was derived to have an energy of $E_{\max }(5000 \mathrm{~km})=2 \times 10^{26} \mathrm{erg}$ (Krucker \& Benz 2000, Table 1). The minimum energy can be derived by equating the total radiation output $P_{\text {tot }}=$ $4.5 \times 10^{5} \mathrm{erg}^{-1} \mathrm{~cm}^{-2} \mathrm{~s}^{-1}$ with the integrated flare energy input. This value was derived by Krucker \& Benz (1998) from the total emission measure observed in coronal EUV lines, averaged over the whole field of view, and includes all radiation losses in the continuum and the lines from UV to X-rays. Solving

$P_{\text {tot }}=\int_{E_{\min }}^{E_{\max }} E \cdot f(E) \mathrm{d} E$

one obtains for $\delta>2$

$E_{\min } \approx\left(\frac{\delta-2}{f_{0}} P_{\text {tot }}\right)^{-\frac{1}{\delta-2}}$. 
Table 1. Observed and derived parameters of nanoflare energy distributions for different height models given by $h, b$ and $\beta$. The temperature used was $T=1.56 \times 10^{6} \mathrm{~K}$.

\begin{tabular}{lrrr}
\hline$h$ & $5000 \mathrm{~km}$ & $500 \mathrm{~km}$ & $\sqrt{(A / 10)}$ \\
\hline$f_{0}$ & $10^{19.2}$ & $10^{18.4}$ & $3.38 \times 10^{11}$ \\
$\delta$ & 2.59 & 2.59 & 2.31 \\
$f_{\mathrm{p} 0}$ & $10^{106.12}$ & $10^{103.6}$ & $1.30 \times 10^{30}$ \\
$\delta_{\mathrm{p}}$ & 6.04 & 6.04 & 3.05 \\
\hline$a$ & $1.8 \times 10^{-5}$ & $4.7 \times 10^{-5}$ & 0.209 \\
$b$ & $5 \times 10^{8}$ & $5 \times 10^{7}$ & 0.145 \\
$c$ & $2.7 \times 10^{14}$ & $1.0 \times 10^{15}$ & $7.91 \times 10^{19}$ \\
$\alpha$ & 0.854 & 0.854 & 0.704 \\
$\beta$ & 0 & 0 & 0.352 \\
$\gamma$ & 1.146 & 1.146 & 0.944 \\
\hline$E_{\min }$ & $2.2 \times 10^{23} \mathrm{erg}$ & $9.8 \times 10^{21} \mathrm{erg}$ & $3.7 \times 10^{20} \mathrm{erg}$ \\
$E_{\mathrm{res}}$ & $9.0 \times 10^{24} \mathrm{erg}$ & $2.8 \times 10^{24} \mathrm{erg}$ & $3.1 \times 10^{24} \mathrm{erg}$ \\
$E_{\max }$ & $2.0 \times 10^{26} \mathrm{erg}$ & $6.3 \times 10^{25} \mathrm{erg}$ & $1.3 \times 10^{26} \mathrm{erg}$ \\
$l_{\min }$ & $1230 \mathrm{~km}$ & $530 \mathrm{~km}$ & $250 \mathrm{~km}$ \\
\hline
\end{tabular}

Here we have used the fact that for our parameter range the upper boundary term of Eq. (27) is vanishingly small. The length of the smallest flares is given by Eqs. (4) and (26)

$l_{\min }=\left(10 \cdot a \cdot E_{\min }^{\alpha}\right)^{1 / 2}$.

If the flare size is equal to the area of a pixel, Eq. (12) shows that the flare energy is equal to the energy in the pixel increase. At this energy, $E_{\text {res }}$, the flare energy distribution will have a low-energy roll-over due to subresolution events. The energy $E_{\text {res }}$ is obtained by solving Eq. (13) for $E=E_{\mathrm{p}} \equiv E_{\text {res }}$. It is consistent with the cutoff energy of $910^{24} \mathrm{erg}$ observed by Krucker \& Benz (1998, Fig. 2, assuming $h=5000 \mathrm{~km})$.

In Fig. 2 the different flare distributions as well as the pixel distribution for $h=\sqrt{(A / 10)}$ are shown for comparison. The distributions are displayed for energies between $E_{\text {res }}$ and $E_{\max }$ as they were observed. The pixel distribution only makes sense for energies higher than the resolution energy, because only these flares cover one or more pixels. This can be illustrated by the following consideration: Let us assume a flare with energy smaller than $E_{\text {res }}$, thus the flare area is smaller than the pixel area. According to Eq. (11), the number of pixel events in the energy interval $\mathrm{d} E_{\mathrm{p}}$ would be smaller than the number of flare-events in the corresponding energy interval $\mathrm{d} E$. This contadicts the assumption that, when any such flare occurs, it has to appear in exactly one pixel. We neglect here the effect of subresolution flares spreading over pixel boundaries. Thus, for flares with area/energy smaller than the resolution, the pixel distribution is equal to the flare distribution and not given by Eq. (11), which in that case makes no sense. This can also be visualized by the definition for the flare distribution, which is the number of flares divided by total observed area, time and energy interval. Obviously, for flares within the observed area this fraction is constant. Reducing the area to the size of a pixel doesn't

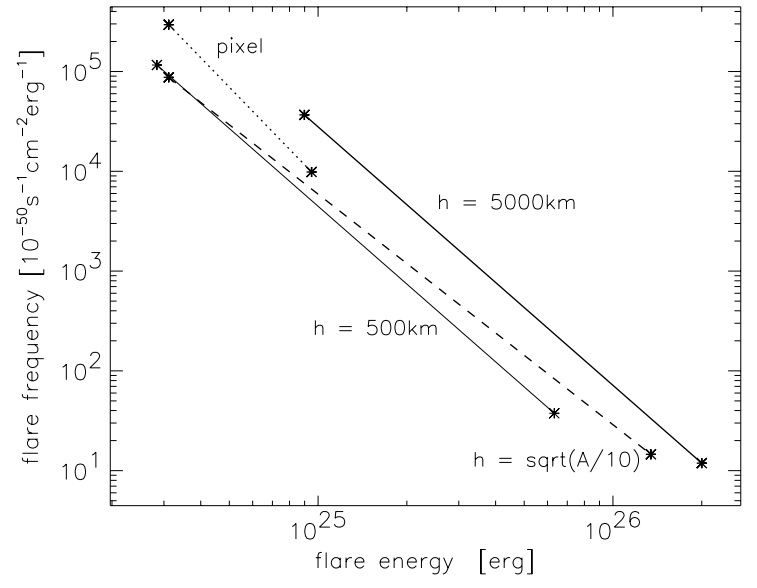

Fig. 2. Various flare distributions and the pixel distribution for $h=\sqrt{A / 10}$ are shown for different height assumptions. The bold line labeled with $h=5000 \mathrm{~km}$ corresponds to the flare energy distribution observed by Krucker \& Benz (1998). The other distributions are derived using Eqs. (16) and (25). The asterisks denote $E_{\text {res }}$ and $E_{\max }$.

change the flare frequency distribution for flares with still smaller size.

\section{Simulation}

In this section we simulate numerically the emission measure variations in individual pixels. The goal of the simulation is to find the conditions for making the simulated pixels resemble the observed emission measure in form and background level, and to make the averaged simulated power spectrum match the observed one in slope and absolute value.

It is assumed that the total emission measure in a pixel at a certain time is given by the sum of the emission measures of all flares brightening at that time. The flares are described by their energy, which is distributed according to the observed pixel distribution for flare energies in the observed range $E_{\text {res }}$ to $E_{\max }$ and flare distribution for the extrapolated sub-resolution events from $E_{\min }$ to $E_{\text {res }}$. The emission measure increase is given by Eq. (3). Because this increase was defined as the difference between a maximum and the preceeding minimum of a brightening, it is about equal to the maximum value of the time dependent emission measure of a flare. The emission measure of a flare increases up to this value and then decreases again according to the flare's time profile.

In the simulation, the flares are randomly distributed in time, according to the flare and pixel distribution. Each flare is defined by its maximum value and the time profile. For every observation, the total emission measure then is the sum of the respective flares' emission measures at that time. To make adequate comparison with the observations, we choose the total observing time $t_{\text {obs }}$ and the equally distributed number of observing points $N_{\text {obs }}$ in the same way as in the observations by Benz \& Krucker (1998). Of the total time dependent emission measure, the 
power spectrum is taken numerically. This process, simulating a pixel and taking the power spectrum, is repeated many times and the resulting power spectra are averaged. The results, the single pixel emission measure level and variations and the average power spectrum, are then compared with the observations.

\subsection{Time profile}

The power spectrum of the emission measure variations in time per pixel depends on the time profile of individual events. Let $\mathcal{M}$ be the emission measure of one flare. The assumed self-similarity of flares requires that $\mathcal{M}$ must depend on the average only on energy in amplitude as well as in duration. The temporal evolution of a flare is given by the rate at which plasma is heated to coronal temperatures, expands and cools. We will assume that the duration is proportional to $E^{s}$. The average emission measure of flares with energy $E$ is therefore

$\mathcal{M}(E, t)=c E^{\gamma} g\left(E^{-s} t\right)$,

where $g$ is the time profile. Note that the maximum value of the emission measure of one large flare is assumed to be the observed emission measure increase. Therefore the time profile $g(x)=g\left(E^{-s} t\right)$ must be chosen in such a way, that $\max (g)=1$. The emission measure increase $\Delta \mathcal{M}=$ $\max (\mathcal{M})=c E^{\gamma}$ then follows the relationship Eq. (3).

Let $\tau(E)$ be the characteristic duration of a flare with thermal energy $E$. We then have

$\tau(E) \propto \frac{E}{\dot{E}} \propto E^{s}$.

The emission measure $\mathcal{M}$ is proportional to the observed energy loss rate from radiation. The corona may also lose energy from conduction at the foot-points of the loop with a rate $Q$. Then $\dot{E} \propto \mathcal{M}+Q$. Assuming that $Q$ follows the same power law as $\mathcal{M}$ or is much smaller and therefore can be neglected, we obtain

$\tau(E) \propto \frac{E}{\Delta \mathcal{M}(E)}$.

Inserting Eq. (3) and comparing with Eq. (31), we immediately obtain $s=1-\gamma$. The simulation results bear out this assumption.

\subsection{Density}

In the derivation of the equation for the thermal energy of a flare (Eq. (1)) it was assumed that the density $n(E)$ is approximately constant in the flare volume. Thus,

$\Delta \mathcal{M}:=\int_{V} n^{2} \mathrm{~d} V \approx n^{2} V$

where $V=A \cdot h=a b E^{\alpha+\beta}$. Solving for $n$ and using Eq. (6), we get

$n=\left(\frac{c}{a b}\right) E^{-s}$.
For $\gamma<1(s>0)$, the density decreases with energy as the duration increases. A physical explanation may be that the longer life time of large flares is partially a result of lower density. Note that the effect is small as $s$ is close to zero in all models. A similar result has been derived by Aschwanden et al. (2000).

\subsection{Results}

In the simulation we use the area dependent height model $h=\sqrt{A / 10}$ with the parameters derived from observations (Table 1, 3rd column). We just remark here that this is not the only height assumption which yields reasonably good results. In particular, the observations are also reproducible for a model with constant height with only minor adjustments in other parameters. Note that the factor $c$, which is directly proportional to the emission measure (Eq. (3)) and therefore proportional to the square root of the power spectrum, is inversely proportional to the temperature squared. Thus, the absolute value of the power spectrum is proportional to the minus fourth power of the temperature. For fine tuning the absolute level of the power spectrum we will adjust the temperature in the simulation. This is equivalent to saying that the effective thermal energy is not given by Eq. (1), but rather by $E=\epsilon 3 k_{\mathrm{B}} T_{\text {obs }} \sqrt{\triangle \mathcal{M A h}}$, with $T_{\text {obs }}$ the observed temperature and $\epsilon$ a correction variable for the true thermal energy. Thus, the temperature we use is $T=\epsilon T_{\text {obs }}$ with $T_{\text {obs }}=1.46 \times 10^{6} \mathrm{~K}$.

The following time profile is used

$g(x)=\left\{\begin{array}{r}\exp (\xi x), \quad x<0 \\ \exp (-\eta x), \quad x \geq 0\end{array}\right.$

with $x=\frac{t-t_{i}}{\tau}, t_{i}$ the peaking time of the $i$ th flare with energy $\stackrel{\tau}{E}$ and duration $\tau=\tau(E)=\left(E / E_{0}\right)^{s} \tau_{0}$, $\tau_{0}=1000 \mathrm{~s}$ the characteristic time of a flare with energy $E_{0}=5.8 \times 10^{25} \mathrm{erg}, s=1-\gamma=0.0556$ and $\xi$ and $\eta$ positive free parameters. For the presented results we have used $\xi=20$ and $\eta=0.8$. The emission measure per pixel is defined as $E M:=\mathcal{M} / A_{\mathrm{p}}$ and is of order of $10^{27} \mathrm{~cm}^{-5}$. The pixel area is $A_{\mathrm{p}}=3.6 \times 10^{16} \mathrm{~cm}^{2}$, and the total observation time is $t_{\mathrm{obs}}=N_{\mathrm{obs}} \Delta t$ with $N_{\mathrm{obs}}=21$ the number of observations and $\Delta t=121.9 \mathrm{~s}$ the time step.

In Fig. 3 some simulated pixels are displayed. The dotted curve is the summed emission measure over the flares in the sub-resolution regime. We see that they are responsible for the background level. The dashed curve occurring occasionally is a resolved nanoflare. The solid curve is the sum of the two: the total emission measure per pixel.

To simulate the effect of noise, the total emission measure at an observed time is distributed randomly within the emission measure noise interval $\mathrm{d} E M$. Figure 4 shows the effect of noise for the same model parameters.

Figure 5 shows the averaged power spectrum of pixels, simulated with the same parameters as in Figs. 3 and 4. The solid curve was observed by Benz \& Krucker (1998) averaged over all pixels, and here corrected in the ordinate. 

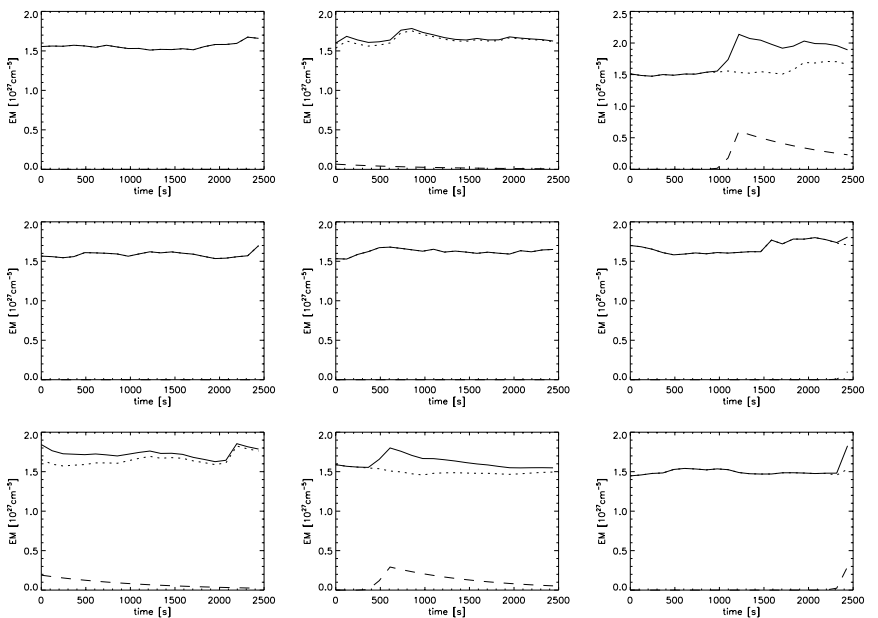

Fig. 3. Emission measure per pixel (solid curve) displayed for random pixels. The dotted curve is background from subresolution flares. The spatially resolved nanoflares are shown by a dashed curve.

The slope of the simulated power spectrum (dotted curve) at low frequencies is mainly given by the choice of the free parameters $\xi$ and $\eta$ (see also Sect. 5). The noise increases the power at all frequencies (dashed-dotted curve). At low frequencies the slope is not changed, but at high frequencies the curve is considerably bent upward, consistent with observations. The absolute values of the power spectrum depend strongly on the temperature as discussed in the beginning of this subsection. The best fit is found to be $T \approx 1.56 \times 10^{6} \mathrm{~K}$, thus $\epsilon \approx 1.068$.

The averaged power spectrum was found to be highly sensitive to the time profile of a flare, which is poorly known from direct observation.

\section{Analytic power spectrum}

In this section the power spectrum of a given nanoflare model distribution is calculated analytically.

Let the expected value of the averaged power spectrum at frequency $\omega$ be $\mathcal{E}\left(\left|\widehat{\mathcal{M}}_{\text {tot }}(\omega)\right|^{2}\right)$, where $\widehat{\mathcal{M}}_{\text {tot }}$ denotes the Fourier transform of the emission measure of a pixel. If the flares follow a Poissonian distribution, are independent of each other and identical at the same energy, it can be shown that the total power spectrum is composed of a flare component and a noise component (see Appendix A)

$\mathcal{E}\left(\left|\widehat{\mathcal{M}}_{\text {tot }}(\omega)\right|^{2}\right)=\mathcal{E}\left(\left|\widehat{\mathcal{M}}_{\mathrm{f}}(\omega)\right|^{2}\right)+\mathcal{E}\left(\left|\widehat{\mathcal{M}}_{\text {noise }}(\omega)\right|^{2}\right)$

with

$\mathcal{E}\left(\left|\widehat{\mathcal{M}}_{\mathrm{fl}}(\omega)\right|^{2}\right)=A_{p} \int_{E_{\min }}^{E_{\max }} f(E)|\widehat{\mathcal{M}}(E, \omega)|^{2} \mathrm{~d} E$

and

$\mathcal{E}\left(\left|\widehat{\mathcal{M}}_{\text {noise }}(\omega)\right|^{2}\right)=\frac{\Delta t}{12}(\mathrm{~d} \mathcal{M})^{2}$,

$\mathrm{d} \mathcal{M}$ being the noise level. Note that in the simulation as well as in the observation the numerical Fourier transform
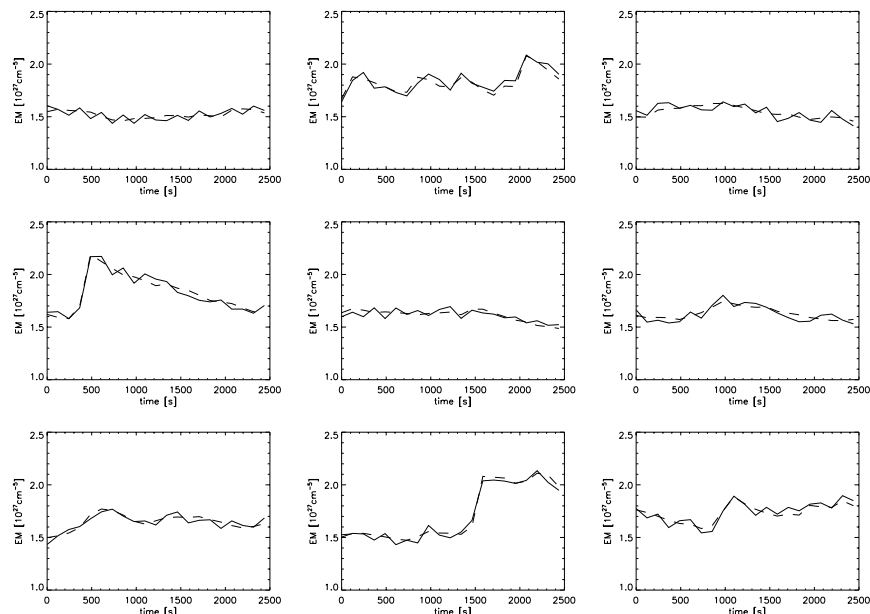

Fig. 4. Noise added emission measure per pixel (solid curve) displayed for random pixels (different from Fig. 3). The dashed curve is the emission measure without noise.

is normalized by division of the total observing time $t_{\mathrm{obs}}$, so here we use the same normalization. Again we consider pixels. Thus the pixel distribution has to be taken for large events, and the integral splits into two parts,

$$
\begin{aligned}
\mathcal{E}\left(\left|\widehat{\mathcal{M}}_{\mathrm{fl}}(\omega)\right|^{2}\right) & =A_{\mathrm{p}}\left[\int_{E_{\mathrm{min}}}^{E_{\mathrm{res}}} f(E)|\widehat{\mathcal{M}}(E, \omega)|^{2} \mathrm{~d} E\right. \\
& \left.+\int_{E_{\mathrm{res}}}^{E_{\max }} f_{\mathrm{p}}\left(E_{\mathrm{p}}\right)\left|\widehat{\mathcal{M}}_{\mathrm{p}}(E, \omega)\right|^{2} \mathrm{~d} E_{\mathrm{p}}\right] .
\end{aligned}
$$

With $f_{\mathrm{p}} \mathrm{d} E_{\mathrm{p}}=A / A_{\mathrm{p}} \cdot f \mathrm{~d} E$ and $\mathcal{M}_{\mathrm{p}}=A_{\mathrm{p}} / A \cdot \mathcal{M}$ we get

$$
\begin{aligned}
\mathcal{E}\left(\left|\widehat{\mathcal{M}}_{\mathrm{fl}}(\omega)\right|^{2}\right) & =A_{\mathrm{p}}\left[\int_{E_{\text {min }}}^{E_{\mathrm{res}}} f(E)|\widehat{\mathcal{M}}(E, \omega)|^{2} \mathrm{~d} E\right. \\
& \left.+\int_{E_{\mathrm{res}}}^{E_{\max }} \frac{A_{\mathrm{p}}}{A(E)} f(E)|\widehat{\mathcal{M}}(E, \omega)|^{2} \mathrm{~d} E\right]
\end{aligned}
$$

The Fourier transform of a single flare is given by

$$
\begin{aligned}
\widehat{\mathcal{M}}(E, \omega) & =c E^{\gamma} g \widehat{(t / \tau)} \\
& =c E^{\gamma} \tau \widehat{g}(\tau \omega)
\end{aligned}
$$

With $g(x)$ from Eq. (35) we have

$\widehat{g}(k)=\frac{\xi+\eta}{(\xi-\mathrm{i} k)(\eta+\mathrm{i} k)}$

with $k=\tau \omega$ and

$$
|\widehat{\mathcal{M}}(E, \omega)|^{2}=c^{2} E^{2 \gamma} \tau(E)^{2} \frac{(\xi+\eta)^{2}}{\left(\xi^{2}+(\tau \omega)^{2}\right)\left(\eta^{2}+(\tau \omega)^{2}\right)} .
$$




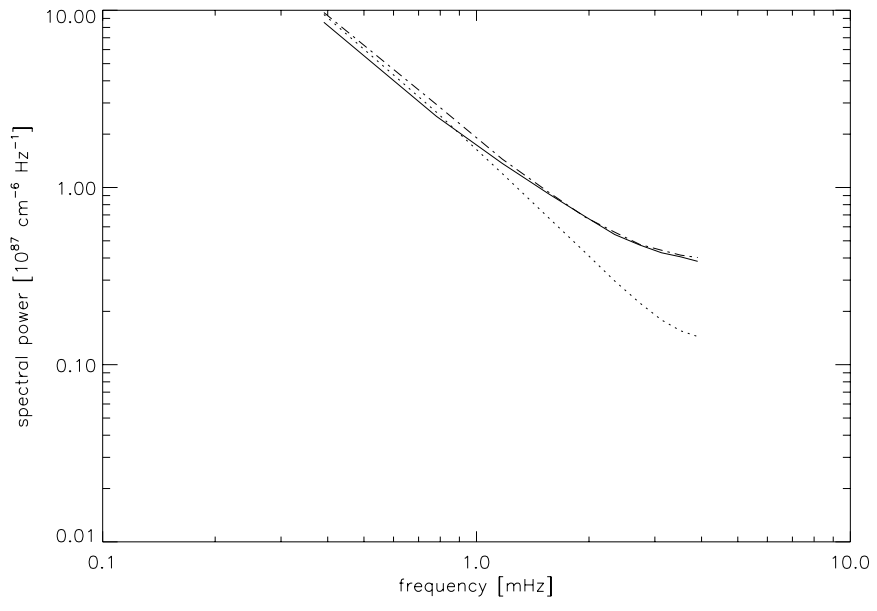

Fig. 5. Simulated power spectrum averaged over 10000 pixels with $\xi=20, \eta=0.8$. The dotted curve is the power spectrum without noise and the dashed-dotted curve the one with noise, the latter is to be compared with the observed power spectrum (solid curve).

Using $\omega=2 \pi \nu$ and inserting all into Eq. (40), we obtain

$\mathcal{E}\left(\left|\widehat{\mathcal{M}}_{\mathrm{fl}}(\nu)\right|^{2}\right)=A_{\mathrm{p}} c^{2} \frac{\tau_{0}^{2}}{E_{0}^{2 s}} f_{0}(\xi+\eta)^{2}$

$$
\begin{aligned}
& \times \int_{E_{\min }}^{E_{\mathrm{res}}} \frac{E^{2-\delta} \mathrm{d} E}{\left(\xi^{2}+\left(2 \pi \tau_{0}\left(\frac{E}{E_{0}}\right)^{s} \nu\right)^{2}\right)\left(\eta^{2}+\left(2 \pi \tau_{0}\left(\frac{E}{E_{0}}\right)^{s} \nu\right)^{2}\right)} \\
&\left.+\frac{A_{\mathrm{p}}}{a} \int_{E_{\mathrm{res}}}^{E_{\max }} \frac{E^{2-\delta-\alpha} \mathrm{d} E}{\left(\xi^{2}+\left(2 \pi \tau_{0}\left(\frac{E}{E_{0}}\right)^{s} \nu\right)^{2}\right)\left(\eta^{2}+\left(2 \pi \tau_{0}\left(\frac{E}{E_{0}}\right)^{s} \nu\right)^{2}\right)}\right]
\end{aligned}
$$

which can be computed numerically. The solution is shown in Fig. 6, dashed curve, with all parameters the same as in Sect. 3.3, in particular $\xi=20$ and $\eta=0.8$. In the dotted curve, aliasing is additionally taken into account. The noise term $\mathcal{E}\left(\left|\widehat{\mathcal{M}}_{\text {noise }}(\omega)\right|^{2}\right)$ is added to the aliased power spectrum (dashed-dotted curve) with $\mathrm{d} \mathcal{M}=A_{\mathrm{p}} \cdot \mathrm{d} E M$. The solid curve shows the observed power spectrum.

\section{Discussion}

It is clear from the previous two sections that a model exists that can explain the emission measure and its fluctuations observed in the quiet solar corona. In this section we address the question of how sensitive the results are to the choice of parameters. Assumptions were made on the height dependence of the thermal energy, extrapolation of the flare distribution to energies several orders of magnitude smaller than the observed range and the shape and energy dependence of the time profile of single pixels. Also, an assumption used throughout this model is the self-similarity of flares at different energies in the average, which is further supported by having found a set of parameters that explains all the available observations. In the following considerations, we will focus on a model with $h=\sqrt{A / 10}$, which seems to be a plausible choice.

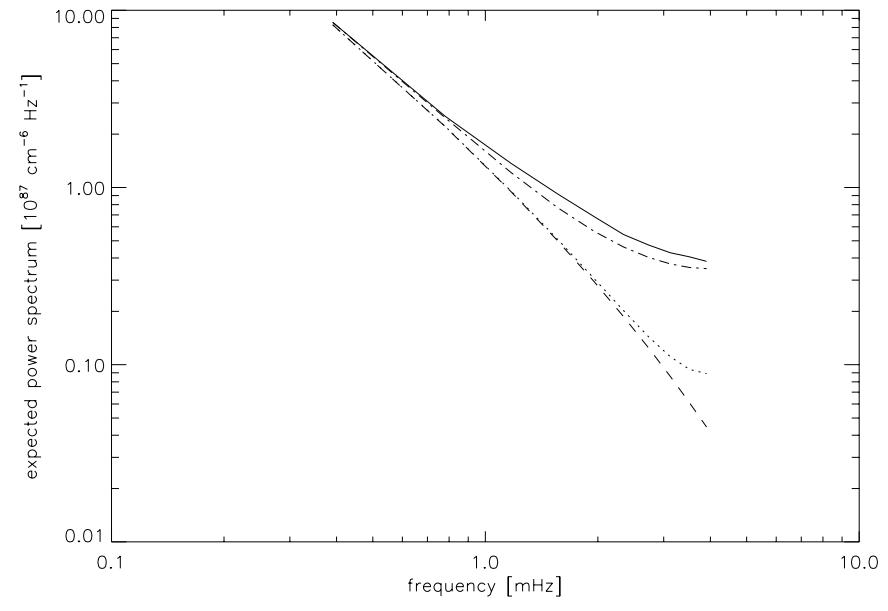

Fig. 6. Analytically calculated power spectrum with $\xi=20$, $\eta=0.8$. The dashed curve shows the true power spectrum, the dotted curve the power spectrum with aliasing and the dasheddotted curve the power spectrum with aliasing and noise. The solid curve is the observed power spectrum.

However, it should be mentioned that this model is not unique in meeting the above requirements. In particular, models with a constant height of $500 \mathrm{~km}$ or $5000 \mathrm{~km}$, respectively, also yield reasonably good results. The remaining free parameters for fitting , $T, \xi$, and $\eta$, however remain practically the same.

The three main observational constraints mentioned in the introduction are the typical emission measure fluctuation of a pixel, its absolute value in emission measure and the shape and value of the power spectrum. As seen in Fig. 3, the sub-resolution flares contribute most to the background level of the emission measure. If we raise the minimum energy or, equivalently, reduce the total input power, the required number of small flares decreases and the background emission level drops drastically. To explain the observed level in emission measure we need to extrapolate the energies to $E_{\min }$. The background level of the preferred model yields values at the low end of the observed emission measure in the quiet corona. A constant emission measure background (e.g. produced by another heating process) could be added without changing the power spectrum.

The nanoflare time profile plays an important role in all three aspects. The background level in emission measure depends on the duration of a flare. A slow increase and decrease in the time profile makes a flare contribute longer to the background, whose level then rises. In this case the fluctuations become smaller. For a fast increase and decrease the background level drops and the fluctuations become more peaked and accentuated.

The rate of change has a strong effect on the slope in the power spectrum. For the time profile given by Eq. (35), the slope of the power spectrum is mainly determined by the free parameters $\xi$ and $\eta$. A slope with the observed value $\kappa=-1.76$ between the lowest two frequency points can be obtained by a wide parameter range, e.g. $(\xi, \eta)=$ $\{(3.41,3.41),(10,1.2),(15,1.0),(20,0.8),(50,0.7)\}$. For a 


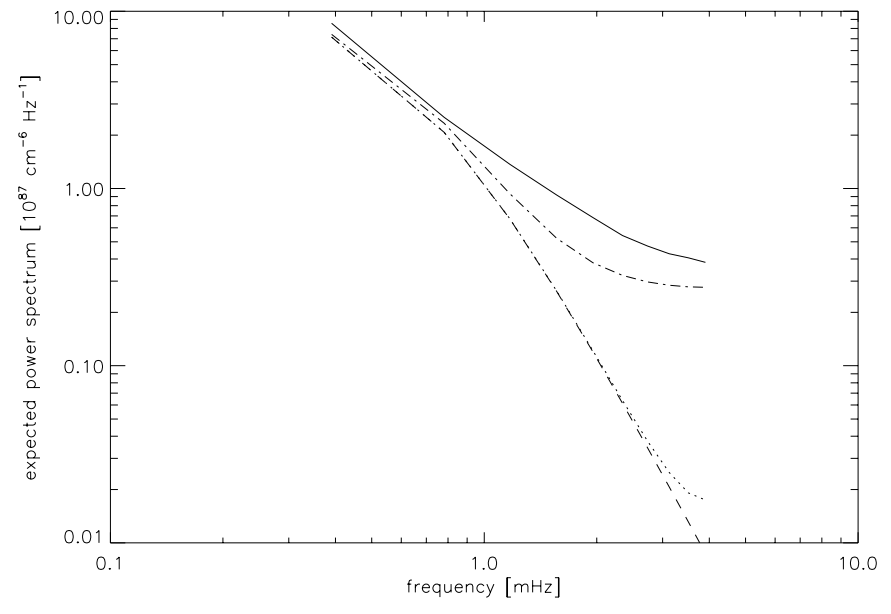

Fig. 7. Analytic power spectrum for a symmetric nanoflare time profile with $\xi=\eta=3.41$. The dashed curve displays the basic power spectrum, the dotted curve the power spectrum with aliasing and the dashed-dotted curve the one with noise and with aliasing. This final calculated curve drops drastically at higher frequencies, not even noise can lift it enough to match the observed spectrum (solid curve) at high frequencies.

symmetric time profile, i.e. $\xi=\eta=3.41$, the power at high frequencies drops so drastically, that a bump appears at medium frequencies that cannot be flattened out by noise (Fig. 7). The more asymmetric the time profile, the straighter is the slope of the power spectrum and the more it resembles the observed one. That the time profile should be so asymmetric is surprising, although it is confirmed immediately by Eq. (45) for the time profile used. If $\xi$ is large compared to the $\nu$ dependence, one bracket-term in the denominator of the integral is almost negligible. If, additionally, $\eta$ is small, the power spectrum is approximately proportional to $\nu^{-2}$. Added noise reduces the steepness of the slope further which then matches the observed $\kappa$. The small discrepancies in the level of the simulated and expected power spectrum come from its sensitive dependence on the parameters $a, b$ and $c$ and numerical errors in the last digits thereof. A small dependence of $\kappa$ also comes from the maximum energy $E_{\max }$. The larger the maximum energy, the steeper the slope.

\section{Conclusion}

Deriving distributions of flare energy and pixel variations from observations requires some assumptions, in particular a model of the effective line-of-sight thickness (height). We have here developed the analytical tools for the transformation between different assumptions published in the literature. These tools are the basis on which we develop a numerical model that simulates the time behavior of individual pixels and nanoflares. It is used here to reproduce the EIT observations of Benz \& Krucker (1998) and Krucker \& Benz (1998).

We have approached the question of nanoflare heating by searching for a model of energy input by nanoflares that can reproduce the three constraints given by the observations of individual pixels: the general appearance of the emission measure variation in time per pixel, the average level of the radiation loss, and the average Fourier power spectrum in time.

We obtain the power spectrum in two different ways, averaging the simulation and calculating the expected value directly. As the evaluation of the integral consumes much less calculation time than the simulation, this method allows us to test systematically different time profiles.

The analysis by analytical and numerical studies has shown that there is at least one such model, assuming self-similarity in energy. Closest to reality may be a model with a height proportional to the square root of the area. The model parameters correspond to the last column in Table 1. To reproduce the observed radiation loss, the observed range of nanoflares (having a lower limit at about $3 \times 10^{24} \mathrm{erg}$, cf. Fig. 2) needs to be extrapolated to lower energies by four orders of magnitude (Table 1). This energy is far below the model suggested by Parker (1983), although there is no stringent theoretical lower limit of magnetic energy release. Nevertheless, the extrapolation is hypothetical for several reasons, in particular the assumption of self-similarity implying a constant power-law index for the distributions in energy, area and height. Other height assumptions, e.g. constant height, also yield reasonable results after slight adjustments for some parameters.

The minimum energy depends on several assumptions. It is larger if the flares introduce other forms of energy into the corona than just the thermal energy of evaporated material, such as fluid motions or wave energy. They would heat the material already existing in the corona and yield a constant background emission measure. Such a background level would reduce the thermal energy input requirements by nanoflares and enhance the minimum energy. A test on the importance of a steady emission measure versus flares could be made by observations of much higher sensitivity than possible today with EIT and TRACE.

A hint of a break-down of our self-similar model may be the small length of flare loops derived (Table 1). A possible remedy may be to assume an energy dependence of the length to width ratio $q$. One may expect from a reconnection scenario that the width and effective height decrease faster with energy than the length, thus forming the smallest flares in thin, long loops. There are currently no observations of this ratio over a sufficient range of flare energies. High resolution observations could clarify this point in the future.

The fitting model is not unique, particularly in the choice of height assumption and average time profile of nanoflares. Nevertheless, we found that the three observational constraints severely limit the range of free parameters once the model assumptions have been made. In particular, the power spectrum is found to be very sensitive to the chosen time profile. As the exact shape of the time profile is not easily observable, this can be used to test how nanoflares evolve and disappear. 
Acknowledgements. We would like to thank S. Krucker for additional information and M. S. Wheatland for providing a first simulation program. We also acknowledge K. W. Smith for suggestions; E. Kraev for mathematical advice; P. Messmer for answering all questions concerning programming; G. Paesold for useful comments and M. Güdel, M. Audard and A. Pauluhn for general discussions and advice. The work at ETH Zurich is financially supported by the Swiss National Science Foundation (grant No. 20-53664.98). We make use of previous EIT/SoHO observations and thank the EIT team, in particular J.-P. Delaboudinière. EIT was funded by CNES, NASA, and the Belgian SPPS.

\section{Appendix A: Derivation of the expected value of the power spectrum}

Consider the observation time interval $t_{\text {obs }}$ divided into $N$ subintervals of equal length through the points $t_{0}=$ $0, t_{1}, \ldots, t_{N}=t_{\text {obs }}$, with $N \gg N_{\text {obs }}$. If the subintervals are chosen to be sufficiently small, we can assume without loss of generality that each flare peaks at one of the points $t_{n}$. Further let us divide our energy range into $M$ equal intervals of length $\Delta E$ through the points $E_{0}=E_{\min }, E_{1}, \ldots, E_{M}=E_{\max }$. Let $p_{m n}$ denote the number of flares of energy between $E_{m}$ and $E_{m}+\Delta E$ peaking at $t_{n}$. For any $m$, let $p_{m n}$ be Poissonian, independent and identically distributed. Their variance is then equal to their expected value and we have

$\operatorname{Var}\left(p_{m n}\right)=\mathcal{E}\left(p_{m n}\right)=\frac{f\left(E_{m}\right) \Delta E}{\int f(E) \mathrm{d} E}$

with $f\left(E_{m}\right)$ the frequency distribution of the flares, normalized to

$\int f(E) \mathrm{d} E=\frac{N}{A_{\mathrm{p}} t_{\mathrm{obs}}}$

Let the emission measure of a flare with energy $E_{m}$ peaking at time $t_{n}$ be

$\mathcal{M}_{m n}(t)=\mathcal{M}\left(E_{m}, t-t_{n}\right)$.

The total emission measure at time $t$ is then given by

$\mathcal{M}_{\mathrm{fl}}(t)=\sum_{m, n} p_{m n} \mathcal{M}_{m n}(t)$

Thus, we have

$\widehat{\mathcal{M}}_{\mathrm{fl}}(\omega)=\sum_{m, n} p_{m n} \widehat{\mathcal{M}}\left(E_{m}, \omega\right) \cdot \mathrm{e}^{-i \omega t_{n}}$,

therefore

$\left|\widehat{\mathcal{M}}_{\mathrm{fl}}(\omega)\right|^{2}=\widehat{\mathcal{M}} \cdot \widehat{\mathcal{M}}^{*}$

$=\sum_{\substack{m_{1}, m_{2} \\ n_{1}, n_{2}}} p_{m_{1} n_{1}} p_{m_{2} n_{2}}$

$\widehat{\mathcal{M}}\left(E_{m_{1}}, \omega\right) \widehat{\mathcal{M}}^{*}\left(E_{m_{2}}, \omega\right) \mathrm{e}^{-i \omega\left(t_{n_{1}}-t_{n_{2}}\right)}$.
As the expected value operator commutes with the sum, we have

$\mathcal{E}\left(\left|\widehat{\mathcal{M}}_{\mathrm{fl}}(\omega)\right|^{2}\right)$

$\begin{array}{rl}=\sum_{\substack{m_{1}, m_{2} \\ n_{1}, n_{2}}} & \mathcal{E}\left(p_{m_{1} n_{1}} p_{m_{2} n_{2}}\right) . \\ & \widehat{\mathcal{M}}\left(E_{m_{1}}, \omega\right) \widehat{\mathcal{M}}^{*}\left(E_{m_{2}}, \omega\right) \mathrm{e}^{-i \omega\left(t_{n_{1}}-t_{n_{2}}\right)}\end{array}$

$=\sum_{\substack{m_{1}, m_{2} \\ n_{1}, n_{2}}}\left[\delta_{m_{1} m_{2}} \delta_{n_{1} n_{2}} \operatorname{Var}\left(p_{m_{1} n_{1}}\right)+\mathcal{E}\left(p_{m_{1} n_{1}}\right) \mathcal{E}\left(p_{m_{2} n_{2}}\right)\right]$

$$
\widehat{\mathcal{M}}\left(E_{m_{1}}, \omega\right) \widehat{\mathcal{M}}^{*}\left(E_{m_{2}}, \omega\right) \mathrm{e}^{-i \omega\left(t_{n_{1}}-t_{n_{2}}\right)} .
$$

Inserting Eq. (A.1)

$\mathcal{E}\left(\left|\widehat{\mathcal{M}}_{\mathrm{fl}}(\omega)\right|^{2}\right)$

$=\frac{A_{\mathrm{p}} t_{\mathrm{obs}}}{N} \sum_{m, n} f\left(E_{m}\right) \widehat{\mathcal{M}}\left(E_{m}, \omega\right) \widehat{\mathcal{M}}^{*}\left(E_{m}, \omega\right) \Delta E$

$+\sum_{m_{1}, m_{2}} f\left(E_{m_{1}}\right) f\left(E_{m_{2}}\right) \widehat{\mathcal{M}}\left(E_{m_{1}}, \omega\right) \widehat{\mathcal{M}}^{*}\left(E_{m_{2}}, \omega\right) \Delta E^{2}$.

$$
\frac{\left(A_{\mathrm{p}} t_{\mathrm{obs}}\right)^{2}}{N^{2}} \sum_{n_{1}, n_{2}} \mathrm{e}^{-\mathrm{i} \omega\left(t_{n_{1}}-t_{n_{2}}\right)}
$$

and taking the sum over $n_{1}, n_{2}$ and $n$, respectively, one obtains

$$
\begin{aligned}
& \mathcal{E}\left(\left|\widehat{\mathcal{M}}_{f l}(\omega)\right|^{2}\right) \\
& =A_{\mathrm{p}} t_{\mathrm{obs}} \sum_{m} f\left(E_{m}\right)\left|\widehat{\mathcal{M}}\left(E_{m}, \omega\right)\right|^{2} \Delta E \\
& +\frac{\left(A_{\mathrm{p}} t_{\mathrm{obs}}\right)^{2}}{N} \cdot \\
& \quad \sum_{m_{1}, m_{2}} f\left(E_{m_{1}}\right) f\left(E_{m_{2}}\right) \widehat{\mathcal{M}}\left(E_{m_{1}}, \omega\right) \widehat{\mathcal{M}}^{*}\left(E_{m_{2}}, \omega\right) \Delta E^{2} .
\end{aligned}
$$

For $N \rightarrow \infty$ the second term vanishes. Taking the limit $\Delta E \rightarrow 0$ we finally obtain

$\mathcal{E}\left(\left|\widehat{\mathcal{M}}_{\mathrm{fl}}(\omega)\right|^{2}\right)=A_{\mathrm{p}} t_{\mathrm{obs}} \int_{E_{\min }}^{E_{\max }} f(E)|\widehat{\mathcal{M}}(E, \omega)|^{2} \mathrm{~d} E$.

White noise adds in the following way. Let the total emission measure with noise be

$\mathcal{M}_{\text {tot }}(t)=\mathcal{M}_{\mathrm{fl}}(t)+\mathcal{M}_{\text {noise }}(t)$

where

$\mathcal{M}_{\text {noise }}(t)=r \mathrm{~d} \mathcal{M}$

with $r$ a random variable between -0.5 and +0.5 and $\mathrm{d} \mathcal{M}$ the noise interval. Then

$$
\begin{aligned}
& \widehat{\mathcal{M}}_{\text {tot }}(\omega)=\widehat{\mathcal{M}}_{\mathrm{fl}}(\omega)+r \widehat{\mathrm{d} \mathcal{M}} \\
& \left|\widehat{\mathcal{M}}_{\text {tot }}\right|^{2}=\left|\widehat{\mathcal{M}}_{\mathrm{f}}\right|^{2}+r^{2}|\widehat{\mathrm{d} \mathcal{M}}|^{2} \\
& +r\left(\widehat{\mathcal{M}}_{\mathrm{fl}} \widehat{\mathrm{d}}^{*}+\widehat{\mathcal{M}}_{\mathrm{fl}}^{*} \widehat{\mathrm{d}} \widehat{\mathcal{M}}\right) \\
& \mathcal{E}\left(\left|\widehat{\mathcal{M}}_{\text {tot }}\right|^{2}\right)=\mathcal{E}\left(\left|\widehat{\mathcal{M}}_{\mathrm{fl}}\right|^{2}\right)+\mathcal{E}\left(r^{2}\right)|\widehat{\mathrm{d} \mathcal{M}}|^{2} \\
& +\mathcal{E}(r)\left(\widehat{\mathcal{M}}_{\mathrm{fl}} \widehat{\mathrm{d}}^{*}+\widehat{\mathcal{M}}_{\mathrm{fl}}^{*} \widehat{\mathrm{d}} \widehat{\mathcal{M}}\right) \text {. }
\end{aligned}
$$


It is $\mathcal{E}(r)=0, \mathcal{E}\left(r^{2}\right)=1 / 12$ and

$$
\begin{aligned}
|\widehat{\mathrm{d} \mathcal{M}}|^{2} & =\sum_{i, j=1}^{N_{\mathrm{obs}}}(\mathrm{d} \mathcal{M})^{2} \mathrm{e}^{-i \omega\left(t_{i}-t_{j}\right)} \Delta t^{2} \\
& =\sum_{i=1}^{N_{\mathrm{obs}}}(\mathrm{d} \mathcal{M})^{2} \Delta t^{2} \\
& =t_{\mathrm{obs}} \Delta t(\mathrm{~d} \mathcal{M})^{2}
\end{aligned}
$$

Thus,

$$
\begin{aligned}
& \mathcal{E}\left(\left|\widehat{\mathcal{M}}_{\text {tot }}(\omega)\right|^{2}\right) \\
& =t_{\text {obs }} \cdot\left[A_{\mathrm{p}} \int f(E)|\widehat{\mathcal{M}}(E, \omega)|^{2} \mathrm{~d} E+\frac{\Delta t}{12}(\mathrm{~d} \mathcal{M})^{2}\right] .
\end{aligned}
$$

\section{References}

Alietti, V., Velli, M., Bocchialini, K., et al. 2000, ApJ, 544, 550 Aschwanden, M. J., Tarbell, T. D., Nightingale, R. W., et al. 2000, ApJ, 535, 1047

Benz, A. O., \& Krucker, S. 1998, Solar Phys., 182, 349

Benz, A. O., \& Krucker, S. 1999, A\&A, 341, 286
Benz, A. O., \& Krucker, S. 2001a, in Recent Insights into the Physics of the Sun and Heliosphere - Highlights from SOHO and Other Space Missions, ed. P. Brekke, B. Fleck, \& J. B. Gurman, ASP Conf. Ser., in press

Benz, A. O., \& Krucker, S. 2001b, ApJ, submitted

Berghmans, D., \& Clette, F. 1999, Solar Phys., 186, 207

Berghmans, D., Clette, F., \& Moses, D. 1998, A\&A, 336, 1039

Brown, J. C., Krucker, S., Güdel, M., \& Benz, A. O. 2000, A\&A, 359, 1185

Cargill, P. J. 1994, ApJ, 422, 381

Gold, T. 1964, in AAS-NASA symposium on the physics of solar flares, ed. W. N. Hess, NASA SP-50, 389

Heyvaerts, J., \& Priest, E. R. 1984, A\&A, 137, 63

Krucker, S., \& Benz, A. O. 1998, ApJ, 501, L213

Krucker, S., \& Benz, A. O. 2000, Solar Phys., 191, 341

Krucker, S., Benz, A. O., Acton, L. W., \& Bastian, T. S. 1997, ApJ, 488, 499

Levine, R. H. 1974, ApJ, 190, 447

Marsh, E., \& Tu, C.-Y. 1997, A\&A, 319, L17

Parker, E. N. 1983, ApJ, 264, 642

Parnell, C. E., \& Jupp, P. E. 2000, ApJ, 529, 554

Ulmschneider, P., Rosner, R., \& Priest, E. R. 1991, Mechanisms of chromospheric and coronal heating (Springer-Verlag, Berlin) 